\title{
Quality of life domains are affected in caregivers of people with Type 2 diabetes: results from a literature review
}

\section{Michele Miranda and Sofia Llahana - London, UK}

Michele Miranda - Community Nurse, Central and North West London - NHS Foundation Trust, email: mimiranda@hotmail.co.uk, telephone: (0044) 7751 935323

Sofia Llahana - Consultant Nurse in Endocrinology, University College London Hospital - NHS Foundation Trust, email:, sofia.llahana@uclh.nhs.uk, telephone (0044) 7989 060871

\section{Background and aim}

Caregivers play a crucial role in self-management of long-term conditions. This aspect is covered in current UK health policies. The aim of this literature review was to explore the impact that Type 2 diabetes has on the caregivers' quality of life [QoL], and its significance in integrated healthcare.

\section{Method}

A search was undertaken in CINAHL, MEDLINE, BNI and ASSIA using the following keywords: carer, caregiver, family/next of $\mathrm{kin} / \mathrm{spouse} / \mathrm{spousal} /$ partner/proxy/diabetes Type 2/quality of life/QoL/health related quality of life/mental quality of life and physical quality of life.

- The inclusion criteria were; articles published in English, in the last 10 years, with adults that explored aspects inherent to caregivers QoL

Five articles satisfied the inclusion criteria for this review (Anaforoğlu et al. 2012, Awadalla et al. 2006, Kovacs et al. 2013, Orvik E. et al. 2006 and Scarton et al. 2014).

The articles were analysed using critical analysis (Booth et al. 2012) and thematic analysis (Polit and Beck 2012).

\section{The search process}

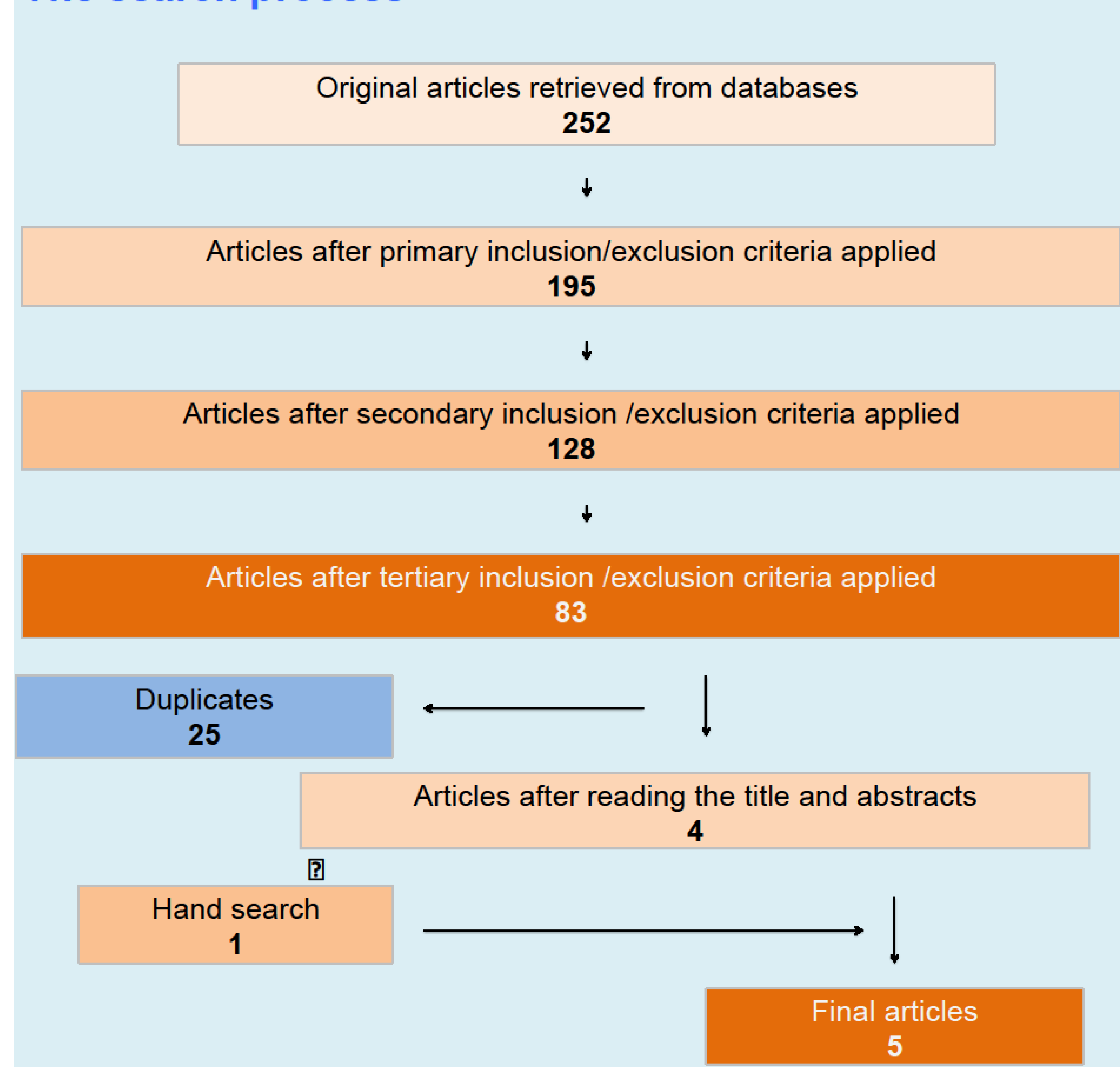

\section{Results}

- The evidence suggests that the overall impact of Type 2 Diabetes in caregivers QOL vary: for some was neutral while for others were positive or negative.

- The emotional wellbeing is the component/domain of caregivers QoL most affected by Type 2 diabetes, and these are reported as depression and anxiety.

- Social functioning and economic burden were other domains affected.

- The majority of the caregivers were female family members.

- Socio demographic and cultural variables appeared to influence caregivers QoL.

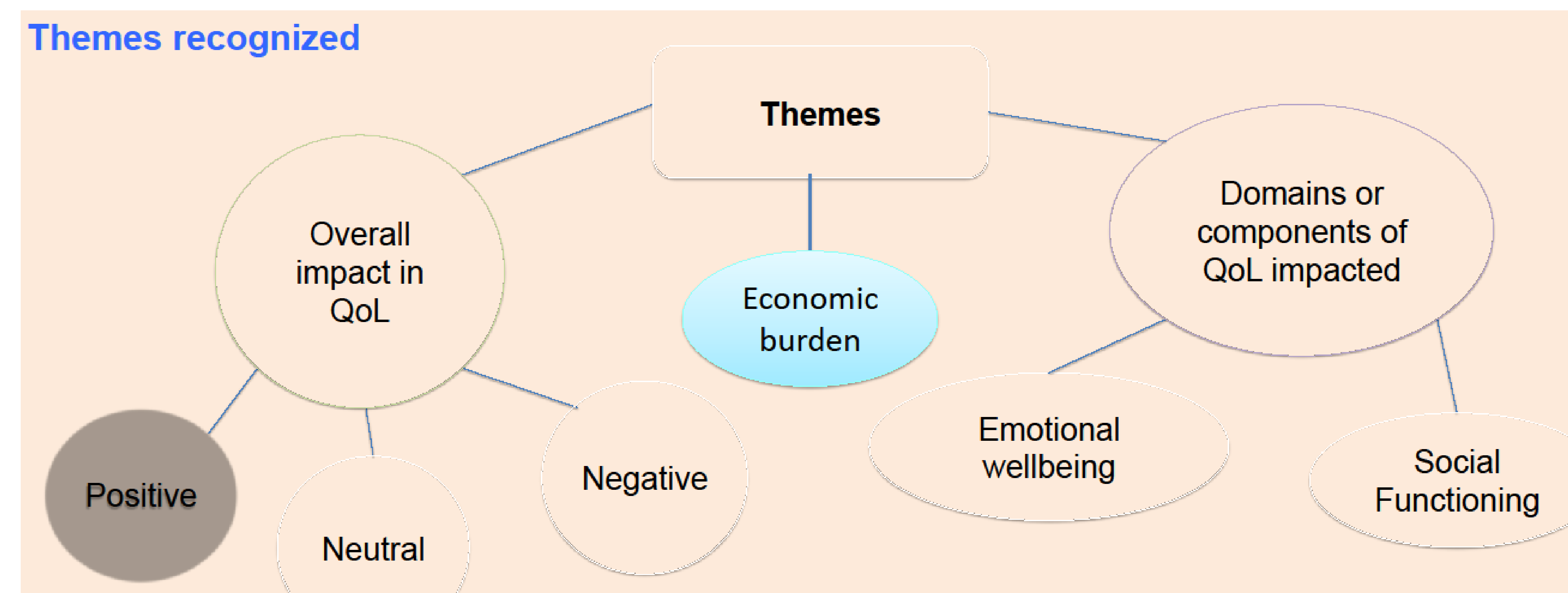

\section{Discussion/lmplications}

- The impact of Type 2 diabetes on caregivers QoL is apparent in an initial overview of the affected QoL domains.

- There is insufficient evidence to ascertain the type of impact of adults' diabetes Type 2 on caregivers' QoL.

- Socio-demographic and cultural differences were identified as variables that can influence caregivers QoL.

- Health care professionals awareness of Type 2 diabetes impact on caregivers' QoL is essential to enable effective integrated healthcare.

\section{Conclusion}

The impact of Type 2 diabetes in caregivers' QoL requires further research, especially to take into consideration socio-demographic and cultural and differences. Educational programmes could be used to raise awareness of healthcare professionals, and to assist them in assessing the impact of Type 2 diabetes on caregivers QoL.

\section{References list}

Anaforoğlu I., Ramazanoğullari I., Algün E. \& Kutanis R. (2012) Depression, anxiety and quality of life of family caregivers of patients with type 2 diabetes. Medical Principles \& Practice 21(4), 360-365. Awadalla A.W., Ohaeri J.U., Al-Awadi S.A. \& Tawfiq A.M. (2006) Diabetes mellitus patients' family caregivers' subjective quality of life. Journal of the National Medical Association 98(5), 727-736. Booth A., Papaioannou D. \& Sutton A. (2012) Systematic Approaches to a Successful Literature Review. Sage Publications Ltd, London.

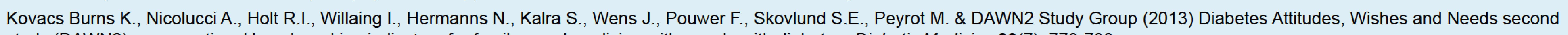
study (DAWN2): cross-national benchmarking indicators for family members living with people with diabetes. Diabetic Medicine 30(7), 778-788.

Orvik E., Johansen O. E., Gullestad L. \& Birkeland K. I. (2006) Health-related quality of life in patients with type 2 diabetes compared to their spouses. European Diabetes Nursing 3(1), 21-26. Polit F. D. \& Beck T. C. (2012) Nursing research: Generating and Assessing Evidence for Nursing Practice. $9^{\text {th }}$ ed. Wotlers Klumer| Lippincott Williams \& Wilkins, London.

Scarton L. J., Bakas T., Poe G. D., Hull M. A., Ongwela L. A. \& Miller W. R. (2014) Needs and Concerns of Family Caregivers of American Indians, African Americans, and Caucasians With Type 2 Diabetes. Clinical Nursing Research pii: 1054773814562879 - Epub ahead of print. (Citation as instructed). 